\title{
Frequência de adesão aos "10 Passos para uma Alimentação Saudável” em escolares adolescentes
}

\author{
Frequency of adherence to the "10 Steps to Healthy Eating" \\ among school-aged adolescents
}

Shanda de Freitas Couto ${ }^{1}$

Samanta Winck Madruga ${ }^{2}$

Marilda Borges Neutzling ${ }^{3}$

Marcelo Cozzensa da Silva ${ }^{4}$

\footnotetext{
${ }^{1}$ Programa de Pós-

Graduação em Educação

Física, Universidade Federal de Pelotas. R. Luiz de Camões 625, Tablada. 96.055-630 Pelotas RS Brasil.

shandacouto@gmail.com

${ }^{2}$ Programa de PósGraduação em Nutrição e Alimentos, Universidade Federal de Pelotas. ${ }^{3}$ Programa de PósGraduação em

Epidemiologia, Universidade Federal do Rio Grande do Sul. ${ }^{4}$ Programa de PósGraduação em Educação Física, Universidade Federal de Pelotas.
}

Abstract The scope of this study was to evaluate the frequency of adherence to "10 Steps to Healthy Eating" of the Ministry of Health in adolescents from high schools in Pelotas in the state of Rio Grande do Sul, Brazil. A school-based crosssectional study was conducted, including 1233 adolescents (13-19 years of age). The frequency of each step was evaluated through a questionnaire on food frequency and other issues included in the "10 Steps." The average number of steps achieved was 1.8. It is noteworthy that none of the subjects achieved entirely all the recommended steps. The highest frequency of compliance was found in step 4, referring to the consumption of the rice and beans. Lower frequencies of adherence were found for step 7 , related to consumption of soft drinks, processed juices, sweets and snacks, and also step 3 on the consumption of fruit/ vegetables/greens. Adherence to the "10 steps to Healthy Eating" by adolescents was very low. Considering the importance of healthy eating in adolescence, greater investment in public policies in the area of food and nutrition is strongly recommended.

Key words Eating habits, Healthy food, Adolescents, Dietary behavior
Resumo O objetivo deste estudo foi avaliar a frequência de adesão aos "10 Passos para uma Alimentação Saudável" do Ministério da Saúde, em adolescentes de escolas de Ensino médio de Pelotas (RS). Foi realizado um estudo transversal de base escolar incluindo 1233 adolescentes de 13 a 19 anos. A frequência de cada passo foi coletada através de um questionário de frequência de consumo de alguns alimentos e outros aspectos contidos nos "10 Passos". A média de passos alcançados foi de 1,8. Ressalta-se que nenhum adolescente aderiu a todos os passos recomendados A maior frequência de adesão foi encontrada no passo 4, referente ao consumo de arroz e feijão; e menores frequência de adesão nos passos 7, referentes ao consumo de refrigerantes, sucos industrializados, doces e guloseimas, e passo 3, relativo ao consumo de Frutas/legumes/hortaliças. A adesão aos "10 passos para uma alimentação saudável" pelos adolescentes foi muito baixa. Considerando a importância de uma alimentação saudável na adolescência, recomenda-se maior investimento em políticas públicas na área de alimentação e nutrição.

Palavras-chave Hábitos alimentares, Alimentação saudável, Adolescentes, Comportamento alimentar 
Introdução

Nas últimas décadas, tem ocorrido aumento significativo na prevalência de doenças crônicas não transmissíveis, como consequência das alterações nos padrões alimentares e de atividade física, associadas ao hábito de fumar e ao consumo de bebidas alcoólicas ${ }^{1,2}$. Evidências indicam aumento na prevalência de excesso de peso e o aparecimento de doenças crônicas em idades cada vez mais precoces ${ }^{2}$. Padrões alimentares inadequados de consumo durante a infância e a adolescência estão ligados não só com a ocorrência da obesidade na juventude ${ }^{2}$, mas também com o risco subsequente de desenvolvimento de doenças como o câncer ${ }^{3}$, diabetes ${ }^{4}$ e doenças cardiovasculares ${ }^{5}$ na idade adulta.

Estudos em diversos países ${ }^{6-8}$ vêm demostrando a baixa adesão de jovens às recomendações nutricionais de seus países. Em 2011, Diethelm et al. ${ }^{6}$ verificaram que adolescentes europeus consomem metade da quantidade recomendada de frutas e legumes e menos de dois terços da quantidade recomendada de leite e derivados. Por outro lado, o mesmo estudo ${ }^{6}$ constatou elevado consumo de carne, gorduras e doces entre os jovens. Resultados semelhantes foram observados na China em 2012, onde apenas 9\%, 14\% e 6\% dos adolescentes pesquisados atingiram os níveis mínimos diários recomendados de ingestão de legumes, frutas, produtos a base de soja e nozes ${ }^{8}$.

No Brasil, apesar do constante investimento do Ministério da Saúde no incentivo à alimentação saudável e do aumento dos níveis de atividade física ${ }^{9,10}$, poucos estudos ${ }^{11}$ avaliaram a adesão a essas recomendações.

Em 2005, o Ministério da Saúde elaborou o Guia Alimentar para a População Brasileira ${ }^{9}$ no âmbito das diretrizes da Política Nacional de Alimentação e Nutrição (PNAN), que se consolidou como elemento brasileiro para implementação das recomendações da Organização Mundial da Saúde ${ }^{12}$, guia esse sintetizado nos "10 Passos para uma Alimentação Saudável" 10 .

Dessa forma, considerando os altos riscos associados à alimentação inadequada na adolescência e a escassez de estudos avaliando a adesão de jovens às recomendações do Ministério da Saúde do Brasil, o presente trabalho teve por objetivo avaliar a frequência de hábitos alimentares saudáveis, baseado nos "10 Passos para uma Alimentação Saudável” e identificar fatores associados a essa adesão em adolescentes matriculados nas escolas de ensino médio da cidade de Pelotas (RS).

\section{Métodos}

Foi realizado um estudo transversal de base escolar com adolescentes de 13 a 19 anos cursando o ensino médio diurno em Pelotas (RS), no período de junho a setembro de 2009. O levantamento de dados abrangeu vários assuntos relacionados à saúde, tais como alimentação, atividade física, doenças crônicas, entre outros.

Todos os 25 estabelecimentos com alunos do ensino médio da cidade de Pelotas foram visitados para estabelecer o número de estudantes, turnos e séries existentes em cada local. Uma das escolas recusou-se a participar do estudo. Dessa forma, 24 escolas (15 instituições estaduais, seis particulares, duas federais e uma municipal) foram incluídas no cálculo amostral. A amostra foi selecionada aletoriamente, com probabilidade proporcional ao tamanho, totalizando 12 escolas para participar do estudo. O cálculo do tamanho amostral foi realizado a partir de dados do estudo piloto onde se trabalhou com a diferença média de pontos (escore dos dez passos) segundo as variáveis independentes. Dentre as variáveis de associação avaliadas, a que necessitou maior tamanho de amostra foi escolaridade da mãe $(\mathrm{n}=324)$, considerando poder de $90 \%$ e nível de confiança de $5 \%$. A este número foram acrescidos $10 \%$ para perdas e recusas e $15 \%$ para controle dos eventuais fatores de confusão, resultando em um total de 410 adolescentes. Também foi realizado o cálculo para a prevalência de alcance dos dez passos, o qual determinou uma amostra de 483 adolescentes. O estudo avaliou um número superior de adolescentes, uma vez que este fez parte de um estudo de consórcio no qual os demais desfechos a serem estudados necessitaram de maior tamanho de amostra para suas avaliações.

Os passos do Ministério da Saúde incluem:

Passo 1 - Faça pelo menos 3 refeições e 2 lanches saudáveis por dia. Não pule as refeições.

Passo 2 - Inclua diariamente 6 porções do grupo do cereais, tubérculos como as batatas e raízes como a mandioca nas refeições. Dê preferência aos grãos integrais.

Passo 3 - Coma diariamente pelo menos 3 porções de legumes e verduras como parte das refeições e 3 porções ou mais de frutas.

Passo 4 - Coma feijão com arroz todos os dias ou, pelo menos, 5 vezes por semana.

Passo 5 - Consuma diariamente 3 porções de leite e derivados e 1 porção de carnes, aves, peixes ou ovos. Retirar a gordura aparente das carnes e a pele das aves antes da preparação. 
Passo 6 - Consuma, no máximo, 1 porção por dia de óleos vegetais, azeite, manteiga ou margarina. Fique atento aos rótulos dos alimentos e escolha aqueles com menores quantidades de gorduras trans.

Passo 7 - Evite refrigerantes e sucos industrializados, bolos, biscoitos doces e recheados, sobremesas e outras guloseimas.

Passo 8 - Diminua a quantidade de sal na comida e retire o saleiro da mesa. Evite consumir alimentos industrializados com muito sal (sódio) como hambúrguer, charque, salsicha, linguiça, presunto, salgadinhos, conservas de vegetais, sopas, molhos e temperos prontos.

Passo 9 - Beba pelo menos 2 litros (6 a 8 copos) de água por dia.

Passo 10 - Pratique pelo menos 30 minutos de atividade física todos os dias e evite as bebidas alcoólicas e o fumo. Mantenha seu peso dentro dos limites saudáveis.

Para a coleta dos dados sobre os passos relativos à alimentação, foi utilizado um quadro que permitiu verificar a frequência de consumo dos alimentos que compõem as recomendações dos 10 Passos para a Alimentação Saudável, utilizando-se o período recordatório de um ano. A pergunta inicial do instrumento foi: Pense sobre sua alimentação no último ano e responda qual o número de vezes, por dia, semana, mês ou ano, que você normalmente come estes alimentos, desde [mês] do ano passado? A seguir uma lista de alimentos era lida ao entrevistado.

A partir do problema encontrado na elaboração do instrumento, que esteve relacionado à subjetividade dos termos utilizados no passo 7 (evitar) e no passo 8 (diminuir), optou-se por utilizar como critério de adesão ao passo os mesmos pontos de corte utilizados por Vinholes et al. ${ }^{11}$ : "evitar" ( 2 vezes ou menos por semana) e “diminuir" (1 vez por semana ou menos).

Para os passos 5, 6 e 8 foram elaboradas questões adicionais ao questionário de frequência para que se alcançasse a adesão de forma integral ao passo. No passo 10, para avaliar o nível de atividade física, aplicou-se um instrumento ${ }^{13}$ com questões sobre a prática de atividades físicas no lazer nos últimos sete dias, e utilizou-se a recomendação de tempo igual ou superior a $300 \mathrm{mi}$ nutos/semana para classificar o adolescente como ativo $^{14}$. No que se refere ao hábito de fumar, considerou-se fumante o adolescente que utilizou pelo menos um cigarro nos últimos 30 dias. A avaliação do consumo de bebidas alcoó- licas foi feita através de uma questão de frequência de consumo de bebidas no último ano, e considerado como positivo, para adesão parcial ao passo 10, a ingestão de bebidas em, no máximo, uma vez por semana. Os adolescentes foram pesados e medidos por nutricionistas treinadas e para avaliação do estado nutricional utilizou-se o índice IMC/idade/sexo e a classificação foi dada a partir da referência da Organização Mundial da Saúde, $2007^{15}$.

O Quadro 1 descreve as categorias utilizadas para verificar a adesão a cada passo. Foi estabelecido que, para o cumprimento do passo, todos os critérios listados deveriam ser atendidos.

As variáveis independentes analisadas foram sexo, idade (anos completos), cor da pele (branco, não branco), nível econômico conforme classificação da Associação Brasileira de Empresas de Pesquisa (ABEP) ${ }^{16}$ [em classes sociais A (maior), B, C, D e E (menor)], escolaridade dos pais (anos completos de estudo), rede de ensino dos adolescentes (federal, estadual, municipal e particular) e autopercepção de saúde (excelente, muito boa, boa, regular ou ruim).

A coleta de dados foi realizada utilizando um questionário autoaplicado testado previamente em estudo piloto, realizado em uma escola não selecionada para o estudo. A aplicação dos questionários foi agendada previamente com as escolas e o questionário preenchido pelo adolescente em sala de aula.

Para a coleta das medidas antropométricas foram utilizados uma balança digital marca SOEHNLE 7755 com capacidade de $150 \mathrm{Kg}$ e precisão de $100 \mathrm{~g}$ e um estadiômetro tipo trena com $200 \mathrm{~cm}$ e precisão de $0,1 \mathrm{~cm}$, ambos sistematicamente calibrados ao longo do trabalho de campo.

Os questionários foram revisados e codificados e, posteriormente, duplamente digitados no programa Epi Info 6.0. A análise dos dados foi realizada no programa estatístico STATA 10.0, e utilizados os recursos da estatística descritiva: média, desvio padrão (DP), frequência relativa e intervalo de confiança 95\%, seguida de análise bivariada através do Teste de Qui-quadrado. O nível de significância adotado foi de $5 \%$.

O presente estudo foi aprovado pelo Comitê de Ética e Pesquisa da Escola Superior de Educação Física da Universidade Federal de Pelotas. O Termo de Consentimento Livre e Esclarecido foi assinado pelos pais ou responsáveis pelos adolescentes. 
Quadro 1. Quadro descritivo das categorias utilizadas para análise da adesão aos "10 Passos para uma Alimentação Saudável”.

\begin{tabular}{|c|c|c|}
\hline Passos & Componentes de cada Passo & $\begin{array}{c}\text { Adesão ao } \\
\text { passo positiva }\end{array}$ \\
\hline $\begin{array}{l}\text { Passo } 1 \text { - Faça pelo menos } 3 \text { refeições e } 2 \text { lanches } \\
\text { saudáveis por dia. Não pule as refeições. }\end{array}$ & Número de refeições & 5 ou + refeições/dia \\
\hline $\begin{array}{l}\text { Passo } 2 \text { - Inclua diariamente } 6 \text { porções do grupo do } \\
\text { cereais, tubérculos como as batatas e raízes como a } \\
\text { mandioca nas refeições. Dê preferência aos grãos } \\
\text { integrais. }\end{array}$ & $\begin{array}{l}\text { Consumo total de cereais, raízes, tubérculos e } \\
\text { integrais (arroz, massa, raízes, pão, bolacha } \\
\text { salgada, cereais integrais) }\end{array}$ & 6 vezes ou $+/$ dia \\
\hline $\begin{array}{l}\text { Passo } 3 \text { - Coma diariamente pelo menos } 3 \text { porções } \\
\text { de legumes e verduras como parte das refeições e } 3 \\
\text { porções ou mais de frutas. }\end{array}$ & $\begin{array}{l}\text { Legumes e hortaliças } \\
\text { Frutas }\end{array}$ & $\begin{array}{l}3 \text { vezes ou }+/ \text { dia } \\
3 \text { vezes ou }+/ \text { dia }\end{array}$ \\
\hline $\begin{array}{l}\text { Passo } 4 \text { - Coma feijão com arroz todos os dias ou, } \\
\text { pelo menos, } 5 \text { vezes por semana. }\end{array}$ & Arroz e Feijão & 5 vezes ou $+/$ semana \\
\hline $\begin{array}{l}\text { Passo } 5 \text { - Consuma diariamente } 3 \text { porções de leite e } \\
\text { derivados e } 1 \text { porção de carnes, aves, peixes ou ovos. } \\
\text { Retirar a gordura aparente das carnes e a pele das aves } \\
\text { antes da preparação. }\end{array}$ & $\begin{array}{l}\text { Carne, aves ou ovos } \\
\text { Leite e derivados } \\
\text { Hábito de retirar a gordura da aparente da } \\
\text { carne e pele de aves }\end{array}$ & $\begin{array}{l}1 \text { vez/dia } \\
3 \text { vezes ou }+/ \text { dia } \\
\text { Sim }\end{array}$ \\
\hline $\begin{array}{l}\text { Passo } 6 \text { - Consuma, no máximo, } 1 \text { porção por dia de } \\
\text { óleos vegetais, azeite, manteiga ou margarina. Fique } \\
\text { atento aos rótulos dos alimentos e escolha aqueles } \\
\text { com menores quantidades de gorduras trans. }\end{array}$ & $\begin{array}{l}\text { Margarina } \\
\text { Utilização de óleos, margarina, manteiga nas } \\
\text { preparações } \\
\text { Leitura dos rótulos dos alimentos } \\
\text { Evita gorduras trans } \\
\text { *(escolha de alimentos com menores } \\
\text { quantidades de gorduras trans) }\end{array}$ & $\begin{array}{l}1 \text { vez ou -/dia } \\
\text { Sim } \\
\text { Sim } \\
\text { Sim }\end{array}$ \\
\hline $\begin{array}{l}\text { Passo } 7 \text { - Evite refrigerantes e sucos industrializados, } \\
\text { bolos, biscoitos doces e recheados, sobremesas e } \\
\text { outras guloseimas. }\end{array}$ & $\begin{array}{l}\text { Refrigerantes } \\
\text { Sucos industrializados } \\
\text { Bolos, bolachas doces ou recheadas } \\
\text { Doces, balas, chocolates e guloseimas }\end{array}$ & $\begin{array}{l}2 \text { vezes ou - / semana } \\
2 \text { vezes ou - / semana } \\
2 \text { vezes ou }-/ \text { semana } \\
2 \text { vezes ou }-/ \text { semana }\end{array}$ \\
\hline $\begin{array}{l}\text { Passo } 8 \text { - Diminua a quantidade de sal na comida e } \\
\text { retire o saleiro da mesa. Evite consumir alimentos } \\
\text { industrializados com muito sal (sódio) como } \\
\text { hambúrguer, charque, salsicha, linguiça, presunto, } \\
\text { salgadinhos, conservas de vegetais, sopas, molhos e } \\
\text { temperos prontos. }\end{array}$ & $\begin{array}{l}\text { Alimentos industrializados: Hambúrguer, } \\
\text { linguiça, charque... } \\
\text { Salgadinhos: Chips ou batata chips } \\
\text { Alimentos enlatados e conservas: ervilha, } \\
\text { milho, pepino... } \\
\text { Hábito de colocar mais sal nas preparações } \\
\text { prontas } \\
\text { Presença de saleiro na mesa }\end{array}$ & $\begin{array}{l}1 \text { vezes ou -/semana } \\
1 \text { vezes ou -/semana } \\
1 \text { vezes ou }-/ \text { semana } \\
\text { Não } \\
\text { Não }\end{array}$ \\
\hline $\begin{array}{l}\text { Passo } 9 \text { - Beba pelo menos } 2 \text { litros ( } 6 \text { a } 8 \text { copos) de } \\
\text { água por dia. }\end{array}$ & Consumo de água & 6 copos ou $+/$ dia \\
\hline $\begin{array}{l}\text { Passo } 10 \text { - Pratique pelo menos } 30 \text { minutos de } \\
\text { atividade física todos os dias e evite as bebidas } \\
\text { alcoólicas e o fumo. Mantenha seu peso dentro dos } \\
\text { limites saudáveis. }\end{array}$ & $\begin{array}{l}\text { Prática de atividade física } \\
\text { Fumo nos últimos } 30 \text { dias } \\
\text { Ingestão de bebidas alcoólicas } \\
\text { Estado Nutricional (IMC/ idade e sexo) }\end{array}$ & $\begin{array}{l}300 \text { minutos/semana } \\
\text { Não } \\
1 \text { vez ou -/semana } \\
\text { Eutrófico }\end{array}$ \\
\hline
\end{tabular}




\section{Resultados}

Dos 1350 adolescentes selecionados para o estudo, foram entrevistados 1233 , perfazendo um total de $8,7 \%$ de perdas ou recusas. A idade média foi de 15,9 anos $(D P=1,2)$ e $54 \%$ dos adolescentes eram do sexo feminino. A maioria da população estudada era de cor da pele branca (79\%) e cerca de $90 \%$ pertenciam às classes econômicas $B$ e C. Quanto à escolaridade dos pais, em torno de $26 \%$ das mães e $23 \%$ dos pais dos adolescentes possuíam mais de 12 anos de estudo (Tabela 1).

A frequência de cada um dos "10 Passos para uma Alimentação Saudável", de acordo com os critérios estabelecidos pelo estudo como marcadores da recomendação atual é apresentada na Tabela 2. O passo que apresentou maior frequência foi o 4 , sendo que $66,5 \%$ dos adolescentes relataram consumir a combinação de arroz e feijão pelo menos cinco vezes por semana. O passo 1 (realizar cinco refeições ou mais por dia) foi o segundo mais frequente com adesão de $24,4 \%$ dos adolescentes. O passo que obteve a menor frequência foi o 7 (consumo de refrigerantes, sucos industrializados, doces e guloseimas, menor ou igual a duas vezes por semana) no qual somente $1,6 \%$ dos adolescentes atingiram a recomendação. Frequência similar foi encontrada no passo 3, referente ao consumo de três porções de legumes ou hortaliças e três de frutas diariamente, sendo alcançada por apenas $1,7 \%$ dos adolescentes.

A média de passos atingidos foi de $1,8(\mathrm{DP}=$ 1,2). A maioria da população atingiu entre um e dois passos $(62 \%)$ e somente $1,7 \%$ atingiu mais de cinco, sendo que o número máximo foi de seis, somente por dois indivíduos $(0,2 \%)$. Ressalta-se que neste estudo nenhum adolescente aderiu a todos os "10 Passos para uma Alimentação Saudável”.

A Tabela 3 analisa a adesão aos 10 passos segundo variáveis de exposição. Em relação ao passo 1 , os resultados mostraram maior adesão por adolescentes do sexo masculino, de classes econômicas mais altas, com pais de maior escolaridade e que apresentam melhor percepção de saúde, com diferenças estatisticamente significativas. A adesão ao passo 2 foi mais frequente entre os meninos $(\mathrm{p}=0,001)$. Ainda, observa-se que as meninas realizavam menor número de refeições diárias (passo 1) e menor consumo de carboidratos (passo 2). No passo 4, verificou-se uma maior adesão por adolescentes de menor nível econômico, de cor da pele não branca, aqueles cujos pais apresentaram menor escolaridade, de maiores idades (17 a 19 anos) e com melhor percepção sobre sua saúde. O passo 5 foi mais frequente entre adolescentes de escolas federais e

Tabela 1. Descrição da amostra de escolares adolescentes cursando ensino médio diurno nas escolas da cidade de Pelotas, Rio Grande do Sul, Brasil ( $\mathrm{n}=1233)$.

\begin{tabular}{|c|c|c|}
\hline Variável & $\mathbf{N}$ & $\%$ \\
\hline \multicolumn{3}{|l|}{ Sexo } \\
\hline Masculino & 567 & 46,0 \\
\hline Feminino & 666 & 54,0 \\
\hline \multicolumn{3}{|l|}{ Idade (anos completos) } \\
\hline $13-14$ & 156 & 12,7 \\
\hline $15-16$ & 678 & 54,9 \\
\hline 17019 & 399 & 32,4 \\
\hline \multicolumn{3}{|l|}{ Cor da pele } \\
\hline Branco & 949 & 79,0 \\
\hline Não Branco & 252 & 21,0 \\
\hline \multicolumn{3}{|l|}{ Nível econômico $(\mathrm{n}=1036)$} \\
\hline A & 82 & 7,9 \\
\hline B & 563 & 54,4 \\
\hline $\mathrm{C}$ & 368 & 35,5 \\
\hline $\mathrm{D}$ & 23 & 2,2 \\
\hline \multicolumn{3}{|c|}{ Escolaridade da mãe(anos completos de estudo) } \\
\hline 0 a 8 anos & 448 & 37,4 \\
\hline 9 a 11 anos & 433 & 36,2 \\
\hline 12 ou mais & 316 & 26,4 \\
\hline \multicolumn{3}{|c|}{ Escolaridade do pai (anos completos de estudo) } \\
\hline 0 a 8 anos & 483 & 42,4 \\
\hline 9 a 11 anos & 389 & 34,2 \\
\hline 12 ou mais & 267 & 23,4 \\
\hline \multicolumn{3}{|l|}{ Escolaridade do adolescente } \\
\hline $1^{\circ}$ ano ensino médio & 470 & 38,1 \\
\hline $2^{\circ}$ ano ensino médio & 397 & 32,2 \\
\hline $3^{\circ}$ ano ensino médio & 366 & 29,7 \\
\hline \multicolumn{3}{|l|}{ Rede de ensino } \\
\hline Federal & 244 & 19,8 \\
\hline Estadual & 695 & 56,4 \\
\hline Municipal & 132 & 10,7 \\
\hline Particular & 162 & 13,1 \\
\hline \multicolumn{3}{|l|}{ Nível de atividade física } \\
\hline Insuficientemente ativos & 788 & 63,9 \\
\hline Suficientemente ativos & 445 & 36,1 \\
\hline \multicolumn{3}{|c|}{ Estado Nutricional (IMC/idade) } \\
\hline Baixo Peso & 9 & 0,7 \\
\hline Eutrófico & 828 & 69,1 \\
\hline Sobrepeso & 267 & 22,3 \\
\hline Obeso & 95 & 7,9 \\
\hline \multicolumn{3}{|l|}{ Tabagismo } \\
\hline Nunca/ex fumante & 1150 & 94,2 \\
\hline Fumante & 71 & 5,8 \\
\hline \multicolumn{3}{|l|}{ Auto-percepção de saúde } \\
\hline Excelente & 233 & 18,9 \\
\hline Muito Boa & 460 & 37,3 \\
\hline Boa & 430 & 34,9 \\
\hline Regular ou Ruim & 110 & 8,9 \\
\hline
\end{tabular}


Tabela 2. Frequência dos 10 Passos da Alimentação Saudável em adolescentes do Ensino Médio da cidade de Pelotas, Rio Grande do Sul, Brasil.

\begin{tabular}{|c|c|c|}
\hline 10 passos & $\%$ & IC95\% \\
\hline $\begin{array}{l}\text { Passo } 1 \\
\quad \text { Número de refeições }\end{array}$ & 24,4 & $22,0-26,8$ \\
\hline $\begin{array}{l}\text { Passo } 2 \\
\text { Consumo total de cereais, raízes, tubérculos e integrais (arroz, massa, } \\
\text { raízes, pão, bolacha salgada, cereais integrais) }\end{array}$ & 21,0 & $18,7-23,4$ \\
\hline $\begin{array}{l}\text { Passo } 3 \\
\quad \text { Legumes e hortaliças } \\
\text { Frutas }\end{array}$ & 1,7 & $1,0-2,5$ \\
\hline $\begin{array}{l}\text { Passo } 4 \\
\quad \text { Arroz e Feijão }\end{array}$ & 66,5 & $63,8-69,1$ \\
\hline $\begin{array}{l}\text { Passo } 5 \\
\text { Carne, aves ou ovos } \\
\text { Leite e derivados } \\
\text { Hábito de retirar a gordura da aparente da carne e pele de aves }\end{array}$ & 2,3 & $1,5-3,3$ \\
\hline $\begin{array}{l}\text { Passo } 6 \\
\text { Margarina } \\
\text { Utilização de óleos, margarina, manteiga nas preparações } \\
\text { Leitura dos rótulos dos alimentos } \\
\text { Evita }^{\star} \text { gorduras trans } \\
\text { ^(escolha de alimentos com menores quantidades de gorduras trans) }\end{array}$ & 23,9 & $21,5-26,4$ \\
\hline $\begin{array}{l}\text { Passo } 7 \\
\text { Refrigerantes } \\
\text { Sucos industrializados } \\
\text { Bolos, bolachas doces ou recheadas } \\
\text { Doces, balas, chocolates e guloseimas }\end{array}$ & 1,6 & $0,9-2,4$ \\
\hline $\begin{array}{l}\text { Passo } 8 \\
\text { Alimentos industrializados: Hambúrguer, linguiça, charque... } \\
\text { Salgadinhos: Chips ou batata chips } \\
\text { Alimentos enlatados e conservas: ervilha, milho, pepino... } \\
\text { Hábito de colocar mais sal nas preparações prontas } \\
\text { Presença de saleiro na mesa }\end{array}$ & 5,2 & $4,0-6,6$ \\
\hline $\begin{array}{l}\text { Passo } 9 \\
\text { Consumo de água }\end{array}$ & 19,2 & $17,0-21,4$ \\
\hline $\begin{array}{l}\text { Passo } 10 \\
\text { Prática de atividade física } \\
\text { Fumo nos últimos } 30 \text { dias } \\
\text { Ingestão de bebidas alcoólicas } \\
\text { Estado Nutricional (IMC/ idade e sexo) }\end{array}$ & 19,4 & $17,2-21,8$ \\
\hline "Todos os 10 passos" & 0 & - \\
\hline
\end{tabular}

com mães de maiores escolaridades. Um maior nível econômico e escolaridade dos pais estiveram associados positivamente ao passo 6 , assim como uma maior frequência desse passo esteve associado a cor da pele branca. O passo 9 foi mais frequente entre os indivíduos não brancos. O sexo masculino e uma melhor percepção de saúde pelos adolescentes estiveram associados à maior frequência do passo 10.

\section{Discussão}

Um aspecto a ser destacado nesse estudo é que a amostra é representativa dos adolescentes de 13 a 19 anos matriculados nas escolas públicas e privadas de Pelotas, e tem poder para avaliar as associações, tendo em vista o cuidadoso processo de seleção, o grande número de entrevistados e o baixo índice de perdas e recusas. Além disso, esse é o primeiro estudo de base escolar que teve por objetivo avaliar a frequência de adesão aos 
Tabela 3. Frequências de adesão dos 10 passos da alimentação saudável segundo as variáveis de exposição estudadas. Pelotas, Rio Grande do Sul, Brasil.

\begin{tabular}{|c|c|c|c|c|c|c|c|c|c|c|}
\hline Variável & $\begin{array}{c}\text { Passo } \\
1 \\
\end{array}$ & $\begin{array}{c}\text { Passo } \\
2 \\
\end{array}$ & $\begin{array}{c}\text { Passo } \\
3 \\
\end{array}$ & $\begin{array}{c}\text { Passo } \\
4 \\
\end{array}$ & $\begin{array}{c}\text { Passo } \\
5 \\
\end{array}$ & $\begin{array}{c}\text { Passo } \\
6 \\
\end{array}$ & $\begin{array}{c}\text { Passo } \\
7 \\
\end{array}$ & $\begin{array}{c}\text { Passo } \\
8 \\
\end{array}$ & $\begin{array}{c}\text { Passo } \\
9 \\
\end{array}$ & $\begin{array}{c}\text { Passo } \\
10 \\
\end{array}$ \\
\hline Sexo [valor $\left.\mathrm{p}^{*}\right]$ & 0,02 & 0,001 & 0,71 & 0,10 & 0,49 & 0,06 & 0,88 & 0,08 & 0,06 & $<0,001$ \\
\hline Masculino & 27,6 & 25,1 & 1,8 & 68,9 & 2,0 & 21,4 & 1,6 & 3,9 & 21,5 & 24,7 \\
\hline Feminino & 21,6 & 17,5 & 1,5 & 64,5 & 2,6 & 26,1 & 1,5 & 6,2 & 17,2 & 15,0 \\
\hline Idade (anos) [valor $\left.\mathrm{p}^{* *}\right]$ & 0,44 & 0,85 & 0,49 & 0,02 & 0,29 & 0,44 & 0,65 & 0,78 & 0,38 & 0,41 \\
\hline $13-14$ & 27,1 & 25,3 & 2,0 & 65,0 & 3,3 & 25,9 & 0 & 3,3 & 22,6 & 22,3 \\
\hline $15-16$ & 24,2 & 19,2 & 1,8 & 63,6 & 2,4 & 22,0 & 2,1 & 5,8 & 16,5 & 19,2 \\
\hline $17-19$ & 23,6 & 22,2 & 1,3 & 72,0 & 1,8 & 26,5 & 1,3 & 4,8 & 22,5 & 18,7 \\
\hline Cor da pele [valor $\left.\mathrm{p}^{*}\right]$ & 0,55 & 0,08 & 0,12 & 0,01 & 0,08 & 0,02 & 0,58 & 0,40 & 0,01 & 0,98 \\
\hline Branco & 25,2 & 20,0 & 1,4 & 64,5 & 2,7 & 25,2 & 1,7 & 5,3 & 17,5 & 19,6 \\
\hline Não branco & 23,4 & 25,2 & 2,8 & 73,2 & 0,8 & 17,9 & 1,2 & 4,0 & 24,9 & 19,5 \\
\hline $\begin{array}{l}\text { Nível socioeconômico } \\
\left.\text { [valor } \mathrm{p}^{* *}\right]\end{array}$ & $<0,001$ & 0,73 & 0,22 & $<0,001$ & 0,20 & $<0,001$ & 0,63 & 0,91 & 0,13 & 0,38 \\
\hline A & 39,0 & 20,0 & 5,0 & 50,6 & 1,2 & 35,9 & 1,2 & 3,8 & 14,8 & 15,2 \\
\hline $\mathrm{B}$ & 25,6 & 21,7 & 1,1 & 62,8 & 2,9 & 26,3 & 1,5 & 5,2 & 17,4 & 22,1 \\
\hline $\mathrm{C}$ & 15,8 & 21,0 & 1,7 & 75,4 & 1,1 & 20,2 & 1,4 & 5,2 & 19,2 & 17,4 \\
\hline $\mathrm{D}$ & 17,4 & 13,6 & 0,0 & 73,9 & 0,0 & 21,7 & 4,2 & 0,0 & 30,4 & 13,6 \\
\hline $\begin{array}{l}\text { Escolaridade da mãe } \\
{\left[\text { valor } \mathrm{p}^{* *}\right]}\end{array}$ & $<0,001$ & 0,44 & 0,37 & $<0,001$ & 0,03 & 0,01 & 1,00 & 0,72 & 0,50 & 0,50 \\
\hline 0 a 8 anos & 18,8 & 23,1 & 1,1 & 73,6 & 1,36 & 21,2 & 1,1 & 5,2 & 19,7 & 19,7 \\
\hline 9 a 11 anos & 25,9 & 18,8 & 1,7 & 68,7 & 2,10 & 23,6 & 2,6 & 5,8 & 18,5 & 20,3 \\
\hline 12 ou mais & 30,8 & 21,2 & 1,9 & 54,0 & 3,83 & 29,6 & 1,0 & 4,5 & 17,8 & 17,5 \\
\hline $\begin{array}{l}\text { Escolaridade do pai } \\
{\left[\text { valor } \mathrm{p}^{* *}\right]}\end{array}$ & $<0,001$ & 0,57 & 0,29 & $<0,001$ & 0,13 & $<0,001$ & 0,33 & 0,69 & 0,22 & 0,71 \\
\hline 0 a 8 anos & 19,1 & 22,3 & 1,3 & 73,5 & 1,7 & 20,6 & 1,3 & 5,6 & 20,6 & 19,2 \\
\hline 9 a 11 anos & 27,6 & 20,7 & 1,8 & 66,9 & 2,9 & 23,1 & 1,3 & 5,2 & 18,1 & 20,8 \\
\hline 12 ou mais & 31,2 & 20,7 & 2,3 & 53,0 & 3,4 & 33,3 & 2,3 & 5,0 & 17,1 & 17,5 \\
\hline $\begin{array}{l}\text { Autopercepção de saúde } \\
{\left[\text { valor }^{* *}\right]}\end{array}$ & 0,03 & 0,20 & 0,40 & 0,047 & 0,10 & 0,96 & 0,69 & 0,29 & 0,69 & $<0,001$ \\
\hline Excelente & 30,0 & 24,6 & 1,7 & 2,0 & 1,7 & 22,1 & 0,4 & 5,2 & 19,3 & 29,1 \\
\hline Muito Boa & 24,4 & 21,0 & 1,5 & 65,8 & 1,8 & 24,8 & 2,9 & 5,9 & 19,5 & 20,4 \\
\hline Boa & 21,7 & 18,8 & 1,0 & 66,2 & 2,6 & 24,7 & 0,2 & 5,2 & 19,6 & 14,1 \\
\hline Regular ou Ruim & 22,7 & 21,7 & 4,7 & 58,9 & 4,6 & 20,8 & 3,7 & 1,9 & 16,4 & 15,1 \\
\hline
\end{tabular}

* Teste do qui-quadrado para heterogeneidade. ${ }^{\star \star}$ Teste do qui-quadrado para tendência linear.

"10 passos para uma Alimentação Saudável” do Ministério da Saúde do Brasil em adolescentes. Uma possível limitação foi o fato de termos considerado que o "porcionamento" dos alimentos seria o número de vezes que o adolescente ingeriu determinado alimento em alguns passos (2, 3, 5 e 6). Essa limitação pode ter resultado em um valor subestimado da frequência de consumo dos alimentos, considerando que, a cada vez que o indivíduo ingeriu determinado alimento, tenha consumido uma porção ou mais.

Os resultados revelaram que o passo 4 (consumo de arroz e feijão) apresentou a maior frequência de adesão. Esse achado corrobora aqueles encontrados na última Pesquisa de Orçamen- tos Familiares-POF 2008-2009 brasileira ${ }^{17}$ que indicou o feijão e o arroz como sendo os alimentos mais consumidos no Brasil. No entanto, essa mesma pesquisa evidencia menor consumo de feijão para adolescentes quando comparados aos adultos e idosos. Outros autores ${ }^{18-21}$ também verificaram em seus estudos que cerca de $60 \%$ dos jovens relatavam consumo semanal regular de feijão. Considerando a importância desse tradicional hábito alimentar brasileiro, principalmente no que se refere ao aporte de fibra alimen$\operatorname{tar}^{22}$, a não adesão por cerca de $30 \%$ dos adolescentes a esse importante passo deve ser olhada com atenção. Estudos recentes ${ }^{23,24}$ enfatizam ainda a importância do consumo regular de arroz e 
feijão como fator protetor a doenças cardiovasculare $^{23}$ e a síndrome metabólica ${ }^{24}$ em adultos. Uma vez que os hábitos alimentares da infância e adolescência tendem a se manter na idade adul$\mathrm{ta}^{25}$ políticas públicas que incentivem o consumo de "feijão com arroz" por adolescentes devem ser permanentemente desenvolvidas.

No que se refere ao passo 1, verificou-se que apenas um quarto dos adolescentes realizava pelo menos cinco refeições diárias. Similarmente, estudo de bases populacional ${ }^{26}$ e escolar ${ }^{18}$ observaram em adolescentes o não seguimento dessa recomendação. Recente estudo de coorte finlandês ${ }^{27}$ mostrou que o padrão de cinco refeições ao dia esteve fortemente associado com o menor risco de sobrepeso/obesidade em adolescentes de 16 anos. Metanálise realizada em $2010^{28}$, analisando 13.998 crianças e adolescentes dos Estados Unidos, Alemanha e Portugal, verificaram em três dos cinco estudos analisados uma redução significativa do risco de obesidade, com o aumento do número de refeições, que persistiu após o ajuste para fatores de confusão, enquanto os dois outros estudos encontraram uma tendência não significativa para a mesma direção. Dada a associação consistente de pular refeições com um risco aumentado de obesidade em crianças e adolescentes, parece prudente promover um padrão regular, com cinco refeições por dia, com composição adequada para as crianças e suas famílias.

A recomendação de evitar o consumo de refrigerantes, doces e guloseimas foi aquela menos seguida pelos adolescentes. No mesmo sentido, estudos nacionais ${ }^{17,20}$ e internacionais ${ }^{6,7}$ têm evidenciado o alto consumo desses alimentos pela população jovem. Analisando dados da Pesquisa Nacional de Nutrição Escolar, Levy et al. ${ }^{19}$ observaram que $92 \%$ dos adolescentes haviam consumido guloseimas pelo menos uma vez na última semana e que cerca de $40 \%$ consumiram refrigerantes mais do que cinco vezes por semana. Em 2008, estudo de revisão mostrou que a chance de crianças tornarem-se obesas aumentava em 1,6 vezes para cada copo adicional de bebida açucarada consumida além de sua ingestão diária habitual. A elevação do consumo desses alimentos caracteriza, em parte, o processo de transição alimentar e nutricional em curso em diversos países ${ }^{1,2}$.

Em relação ao consumo de sal e alimentos industrializados, constatou-se que apenas uma pequena parcela dos adolescentes aderiu totalmente à recomendação. Embora a maioria dos jovens tenha relatado não adicionar sal aos alimentos já preparados, estudos recentes ${ }^{29,30}$ indicam que grande parte do sal ingerido vem de alimentos processados. Uma metanálise de ensaios controlados ${ }^{29}$ mostrou que a ingestão excessiva de sal na dieta afeta a pressão arterial mesmo em crianças e adolescentes. Em 2011, Libuda et al. ${ }^{30}$ verificaram ainda que elevada ingestão de alimentos processados, ricos em sal, teria impacto negativo na composição corporal de crianças e adolescentes, independente de seu consumo de bebidas açucaradas.

Quanto à ingestão diária de verduras, legumes e frutas (passo 3), observou-se frequência de consumo muito baixa entre os jovens. Similarmente dados nacionais indicam que menos de $10 \%$ da população atinge tais recomendações para consumo de frutas, verduras e legumes ${ }^{17}$. Um estudo com escolares do $9^{\circ}$ ano de capitais brasileiras $^{19}$ evidenciou frequência de consumo inferior a uma vez por semana de frutas e hortaliças por mais de $20 \%$ dos jovens estudados. Outros autores ${ }^{20,31}$ têm encontrado resultados semelhantes, mostrando adolescentes consumindo frutas, verduras e legumes em quantidades e frequências muito aquém das recomendações. A Organização Mundial da Saúde ${ }^{2}$ vem sistematicamente alertando os países sobre a importância do consumo de pelo menos 400g diárias desses alimentos, considerando seu comprovado papel protetor em relação às doenças crônicas não transmissíveis.

Analisando a frequência total de adesão aos "10 passos para uma Alimentação Saudável" na população adulta de Pelotas, estudo ${ }^{11}$ encontrou que apenas $1,1 \%$ dos indivíduos seguiram todos os passos integralmente, e apresentaram, em média, adesão de seis passos. Os resultados do presente estudo mostram uma situação ainda mais preocupante, visto que nenhum adolescente aderiu a todos os passos, e a média de adesão foi de 1,8 passos. Esses resultados são alarmantes diante do aumento da prevalência de sobrepeso e obesidade na adolescência e por ser este um período de formação e consolidação dos hábitos alimentares.

$\mathrm{Na}$ análise estratificada, observou-se que poucos passos apresentaram diferenças estatisticamente significativas em relação às variáveis analisadas. A maior adesão às recomendações parece estar associada a adolescentes do sexo masculino, de maior nível econômico, com pais de maior escolaridade e àqueles jovens com melhor autopercepção sobre sua saúde. A exceção ficou por conta do passo 4 (consumo regular de feijão e arroz), que foi mais frequente em adolescentes de menor nível socioeconômico e com pais menos escolarizados. 

corporal frequentemente evidenciada entre adolescentes do sexo feminino ${ }^{32}$.

No que diz respeito ao nível econômico, os adolescentes mais ricos omitiram menos refeições e ingeriram com maior frequência alimentos ricos em gorduras. Aqueles de menor nível econômico ingeriam mais feijão com arroz. Similarmente, o estudo de Neutzling et al. ${ }^{20}$ verificou que a frequência de consumo diário de feijão nos jovens de menor nível econômico foi quase o dobro daquela observada nos mais ricos. A mesma tendência foi verificada na POF 2008-2009 ${ }^{17}$, na qual foi observado consumo maior de itens alimentares considerados saudáveis, como feijão e preparações à base de feijão nas faixas de menor renda. Enquanto que esta mesma pesquisa ${ }^{17}$ evidenciou uma relação positiva entre maior renda familiar e consumo de alguns itens alimentares, tais como leite e derivados, frutas, verduras, e refrigerantes.

Dados da PeNSE ${ }^{19}$ indicam uma associação entre menor escolaridade da mãe e maior consumo de feijão entre adolescentes. Esses resultados mostram que hábitos alimentares de adolescentes sofrem uma grande influência do nível econômico das famílias, assim como aspectos culturais nas escolhas dos alimentos.

Uma melhor autopercepção de saúde esteve associada a uma menor omissão de refeições, maior consumo de arroz e feijão e a uma maior

frequência de adesão ao passo 10, que se refere a menores prevalências de excesso de peso, de sedentarismo e de consumo de fumo e álcool. Esses resultados sugerem a relação entre comportamentos saudáveis, como prática de atividade física, alimentação e saúde na vida dos indivíduos. Resultados semelhantes foram encontrados em um estudo realizado nos Estados Unidos em 2007: tabagismo, sedentarismo e práticas alimentares inadequadas estiveram diretamente associados a um consumo mais frequente de fast foo $d^{33}$. Estudo nacional com escolares do $9^{\circ}$ ano do ensino fundamental, verificou uma associação negativa entre tabagismo e comportamentos saudáveis, tais como a prática de atividade física ${ }^{34}$.

\section{Conclusão}

Conclui-se que a frequência de hábitos alimentares saudáveis nos escolares adolescentes de Pelotas foi muito baixa, salientando-se que nenhum jovem aderiu totalmente aos "10 Passos para uma alimentação Saudável". Considerando a complexidade da determinação econômica, psicológica, cultural dos hábitos alimentares é necessário maior investimento em políticas públicas que visem intervir nos determinantes desses hábitos. A ação intersetorial da área de produção de alimentos, educação nutricional e atividade física, além do controle de propagandas que incentivem o consumo de alimentos pouco saudáveis, e uma maior divulgação das recomendações sobre alimentação entre os adolescentes, poderiam contribuir substancialmente para uma maior adesão da população aos 10 passos preconizados pelo Ministério da saúde do Brasil. 


\section{Colaboradores}

SF Couto, SW Madruga, MB Neutzling e MC Silva participaram das seguintes etapas de elaboração do manuscrito: concepção, delineamento, análise e interpretação dos dados; e redação do artigo.

\section{Agradecimentos}

Este estudo contou com suporte da Fundação de Amparo a Pesquisa do Rio Grande do Sul- FAPERGS que contribuiu através de Bolsa de Iniciação Científica para a coleta e processamento dos dados.

\section{Referências}

1. Popkin BM Adair LS Ng SW. Global nutrition transition and the pandemic of obesity in developing countries. Nutr Rev 2012; 70(1):3-21.

2. World Health Organization (WHO), Food and Agriculture Organization (FAO). Expert Consultation on Diet, and Nutrition and the prevention diseases. Diet Nutrition and the Prevention of chronic diseases: report of a joint WHO/FAO WHO expert consultation. Geneva: WHO, FAO; 2003. (WHO Technical Report Series, n. 916)

3. Mahabir S. Association Between Diet During Preadolescence and Adolescence and Risk for Breast Cancer During Adulthood. J Adolesc Health 2012; 52(Supl. 5):S30-S35.

4. Malik VS, Fung TT, van Dam RM, Rimm EB, Rosner B, Hu FB. Dietary patterns during adolescence and risk of type 2 diabetes in middle-aged women. Diabetes Care 2012; 35(1):12-18.

5. Ness AR, Maynard M, Frankel S, Smith GD, Frobisher C, Leary SD, Emmett PM, Gunnell D. Diet in childhood and adult cardiovascular and all cause mortality: the Boyd Orr cohort. Heart 2005; 91(7): 894-898.

6. Diethelm K, Jankovic N, Moreno LA, Huybrechts I, De Henauw S, De Vriendt T, González-Gross M, Leclercq C, Gottrand F, Gilbert CC, Dallongeville J, Cuenca-Garcia M, Manios Y, Kafatos A, Plada M, Kersting M; HELENA Study Group. Food intake of European adolescents in the light of different food-based dietary guidelines: results of the HELENA (Healthy Lifestyle in Europe by Nutrition in Adolescence) Study. Public Health Nutr 2012; 15(3): 386-398.

7. Sebastian RS, Wilkinson Enns C, Goldman JD. US adolescents and MyPyramid: associations between fast-food consumption and lower likelihood of meeting recommendations. J Am Diet Assoc 2009; 109(2):226-235.

8. Zhang CX, Chen YM, Chen WQ, Su YX, Wang CL, $\mathrm{Wu}$ JN. Food group intake among adolescents in Guangzhou city compared with the Chinese dietary guidelines. Asia Pac J Clin Nutr 2012; 21(3):450-456.

9. Brasil. Ministério da Saúde (MS). Coordenação Geral da Política de Alimentação e Nutrição. Guia alimentar para a população brasileira 2005, Brasília: MS. 210p. [Internet]. [acessado 2014 abr 6]. Disponível em: http://dtr2001.saude.gov.br/editora/produtos/livros/pdf/05_1109_M.pdf.

10. Brasil, Ministério da Saúde (MS). Coordenação Geral da Política de Alimentação e Nutrição. Alimentação saudável para todos: siga os 10 passos. [Internet]. [acessado 2013 abr 7]. Disponível em: http://189.28. 128.100/nutricao/docs/geral/10passosAdultos.pdf

11. Vinholes DB, Assuncao MC, Neutzling MB. Frequency of healthy eating habits measured by the 10 Steps to Healthy Eating score proposed by the Ministry of Health: Pelotas, Rio Grande do Sul State, Brazil. Cad Saude Publica 2009; 25(4):791-799. 
12. Barreto SM, Pinheiro ARO, Sichieri R, Monteiro CA, Bastista Filho M, Schimidt MI, Lotufo P, Assis AM, Guimarães V, Recine EGIG, Victora CG, Coitinho D, Passos VMA. Análise da Estratégia Global para Alimentação, Atividade Física e Saúde, da Organização Mundial da Saúde. Epidemiologia e Serviços de Saúde 2005; 14(1):41-68.

13. Bastos JP, Araujo CL, Hallal PC. Prevalence of insufficient physical activity and associated factors in Brazilian adolescents. J Phys Act Health 2008; 5(6):777-794.

14. Dobbins M, De Corby K, Robeson P, Husson H, Tirilis D. School-based physical activity programs for promoting physical activity and fitness in children and adolescents aged 6-18. Cochrane Database Syst Rev 2009(1):CD007651.

15. De Onis M, Onyango AW, Borghi E, Siyam A, Nishida C, Siekmann J. Development of a WHO growth reference for school-aged children and adolescents. Bull World Health Organ 2007; 85(9):660-667.

16. Associação Brasileira de Empresas de Pesquisa (ABEP). Critério de classificação econômica Brasil. 2008. [Internet]. [acessado 2013 set 2]. Disponível em: http://www.abep.org/codigosguias/CCEB2008Base2006e2007.pdf

17. Instituto Brasileiro de Geografia e Estatística (IBGE). Coordenação de Trabalho e Rendimento. Pesquisa de Orçamentos Familiares 2008-2009: Análise do Consumo Alimentar Pessoal no Brasil. Rio de Janeiro: IBGE; 2011.

18. Dalla Costa M, Cordoni Júnior L, Matsuo T. Hábito alimentar de escolares adolescentes de um município do oeste do Paraná. Rev Nutrição 2007; 20(5):461-471.

19. Levy RB, Castro IR, Cardoso Lde O, Tavares LF, Sardinha LM, Gomes Fda S, Costa AW. Food consumption and eating behavior among Brazilian adolescents: National Adolescent School-based Health Survey (PeNSE), 2009. Cien Saude Colet 2010; 15(Supl. 2):3085-3097.

20. Neutzling MB, Assunção MCF, Malcon MC, Hallal PC, Menezes AMB. Food habits of adolescent students from Pelotas, Brazil. Rev Nutrição 2010; 23(3): 379-388.

21. Malta DC, Sardinha LMV, Mendes I, Barreto SM, Giatti L, Castro IRR, Moura L, Dias AJR, Crespo C. Vigilância de fatores de risco e proteção de doenças crônicas e não transmissíveis em adolescentes no Brasil. Cien Saude Colet 2010; 15(Supl. 2):3009-3019.

22. Trinidad TP, Mallillin AC, Loyola AS, Sagum RS, Encabo RR. The potential health benefits of legumes as a good source of dietary fibre. $\mathrm{Br} J \mathrm{Nutr}$ 2010; 103(4):569-574.

23. Mattei J, Hu FB, Campos H. A higher ratio of beans to white rice is associated with lower cardiometabolic risk factors in Costa Rican adults. Am J Clin Nutr 2011; 94(3):869-876.
24. Noel SE, Newby PK, Ordovas JM, Tucker KL. A traditional rice and beans pattern is associated with metabolic syndrome in Puerto Rican older adults. $J$ Nutr 2009; 139(7):1360-1367.

25. Mikkila V, Räsänen L, Raitakari OT, Pietinen P, Viikari J. Longitudinal changes in diet from childhood into adulthood with respect to risk of cardiovascular diseases: The Cardiovascular Risk in Young Finns Study. Eur J Clin Nutr 2004; 58(7):1038-1045.

26. Madruga SW, Araujo CL, Bertoldi AD. Frequency of fiber-rich food intake and associated factors in a Southern Brazilian population. Cad Saude Publica 2009; 25(10):2249-2259.

27. Jaaskelainen A, Schwab U, Kolehmainen M, Pirkola J, Järvelin MR, Laitinen J. Associations of meal frequency and breakfast with obesity and metabolic syndrome traits in adolescents of Northern Finland Birth Cohort 1986. Nutr Metab Cardiovasc Dis 2012; 23(10):1002-1009.

28. Koletzko B, Toschke AM. Meal patterns and frequencies: do they affect body weight in children and adolescents? Crit Rev Food Sci Nutr 2010; 50(2):100-105.

29. He FJ, MacGregor GA. Importance of salt in determining blood pressure in children: meta-analysis of controlled trials. Hypertension 2006. 48(5):861869.

30. Libuda L, Kersting M, Alexy U. Consumption of dietary salt measured by urinary sodium excretion and its association with body weight status in healthy children and adolescents. Public Health Nutr 2012; 15(3):433-441.

31. Rieth MA, Moreira MB, Fuchs FD, Moreira LB, Fuchs SC. Fruits and vegetables intake and characteristics associated among adolescents from Southern Brazil. Nutr J 2012; 11:95.

32. Andrade RG, Pereira RA, Sichieri R. Food intake in overweight and normal-weight adolescents in the city of Rio de Janeiro. Cad Saude Publica 2003. 19(5):1485-1495.

33. Larson NI, Story M, Perry CL, Neumark-Sztainer D, Hannan PJ. Are diet and physical activity patterns related to cigarette smoking in adolescents? Findings from Project EAT. Prev Chronic Dis 2007; 4(3): A51.

34. Barreto SM, Giatti L, Casado L, Moura L, Crespo C, Malta DC. Exposição ao tabagismo entre escolares no Brasil. Cien Saude Colet 2010; 15(Supl. 2):30273034.

Artigo apresentado em 30/10/2013

Aprovado em 08/11/2013

Versão final apresentada em 12/11/2013 